\title{
RESOLUCIÓN DE UN CASO DE PRIAPISMO ARTERIAL SECUNDARIO A FISTTULA ARTERIOCAVERNOSA BILATERAL MEDIANTE EMBOLIZACIÓN SELECTIVA CON MATERIAL REABSORBIBLE
}

\author{
I. CASTAÑO GONZÁLEZ, I. MONCADA IRIBARREN, D. SUBIRÁ RÍOS, \\ M. MORALEJO GÁRATE, J.I. MARTÍNEZ SALAMANCA, R. CABELLO BENAVENTE, \\ C. HERNÁNDEZ FERNÁNDEZ
}

Servicio de Urología. Hospital General Universitario Gregorio Marañón. Madrid. España.

Actas Urol Esp. 28 (10): 777-780, 2004

\section{RESUMEN}

RESOLUCIÓN DE UN CASO DE PRIAPISMO ARTERIAL SECUNDARIO A FÍSTULA ARTERIOCAVERNOSA BILATERAL MEDIANTE EMBOLIZACIÓN SELECTIVA CON MATERIAL REABSORBIBLE

El priapismo se define como la persistencia de erección no resultante del deseo sexual. Las nuevas clasificaciones distinguen entre priapismo veno-oclusivo, de bajo flujo o isquémico y priapismo arterial, de alto flujo o no isquémico. El trauma perineal o peneano son los responsables de la mayoría de los casos de priapismo arterial, creándose una fístula arteriocavernosa. El diagnóstico viene dado por la clínica, confirmándose a través de gasometría por punción de cuerpos cavernosos y la ecografía doppler color. La arteriografía con embolización selectiva, constituye un método terapéutico seguro y efectivo.

Presentamos el caso de un priapismo arterial secundario a fístula arteriocavernosa bilateral, resuelto mediante la embolización selectiva con material reabsorbible.

PALABRAS CLAVE: Priapismo arterial. Angiografia. Embolización.

\section{ABSTRACT \\ RESOLUTION ONE CASE ARTERIAL PRIAPISM SECONDARY TO BILATERAL ARTERIOCAVERNOUS FISTULA WITH SELECTIVE EMBOLIZATION USING REABSORBIBLE MATERIAL}

Priapism is defined as persistent erection without sexual stimulus. The new classifications make a distinction between venooclusive low flow priapism, isquemic and arterial high flow priapism, non isquemic. The perineal or penis trauma are responsible the most of cases arterial priapism, formation of an arteriocavernous fistula. The diagnosis is clinical, blood gas analysis and colour Doppler ultrasonography of the corpora cavernosa confirmed the diagnosis. The arteriography with selective embolization constitutes a safe and effective therapeutic method.

We report one case of arterial priapism secondary to bilateral arteriocavernous fistula resolved with selective embolization using reabsorbible material. 
$\mathrm{E}$ priapismo arterial es una entidad poco usual, caracterizada por la persistencia de erección continuada, sin guardar relación con el deseo sexual. A diferencia de la erección fisiológica, el estado de erección está limitado a los cuerpos cavernosos con flacidez del cuerpo esponjoso ${ }^{1,3}$.

El trauma perineal cerrado o bien peneano, son los responsables del desencadenamiento del priapismo arterial o de alto flujo, caracterizado por la instauración de una erección incompleta, e indolora en la mayoría de los casos, secundaria a la creación de una fístula arteriocavernosa unilateral, o bien bilateral como en el caso que pasaremos a describir a continuación.

El diagnóstico del priapismo arterial, viene dado por la historia clínica y exploración física en la mayor parte de los pacientes. La gasometría obtenida por punción directa de los cuerpos cavernosos y la realización de una ecografía Doppler color, nos servirán como pruebas complementarias que confirmen nuestra sospecha diagnóstica. La realización de una arteriografía se empleará con fines diagnósticos y terapéuticos en el priapismo arterial, procediéndose a la embolización selectiva de la fístula originada ${ }^{1-7}$.

\section{CASO CLÍNICO}

Presentamos el caso de un varón de 25 años, sin antecedentes personales de interés.

Es atendido en el Servicio de Urgencias por traumatismo cerrado en el pene en flaccidez, ocasionado por golpe directo contra el canto de una puerta, con aparición a las 48 horas de erección no dolorosa, incompleta, que aumenta con el estímulo sexual y durante erecciones nocturnas.

La exploración muestra un pene normal, en erección incompleta, no doloroso a la palpación. No se objetivan signos de traumatismo (ausencia de heridas, hematomas,...). Los testes y escroto son normales.

Se procedió a la realización de una gasometría de sangre obtenida por punción del cuerpo cavernoso, mostrando un patrón sanguíneo de tipo arterial, con una $\mathrm{pO}_{2}$ de $97 \mathrm{mmHg}$ y una saturación de oxígeno del $98 \%$.

Ante la alta sospecha diagnóstica de priapismo arterial, se realizó ecografía Doppler color peneana (Fig. 1) que muestra intenso flujo arterial en raíz de ambos cuerpos cavernosos. Se

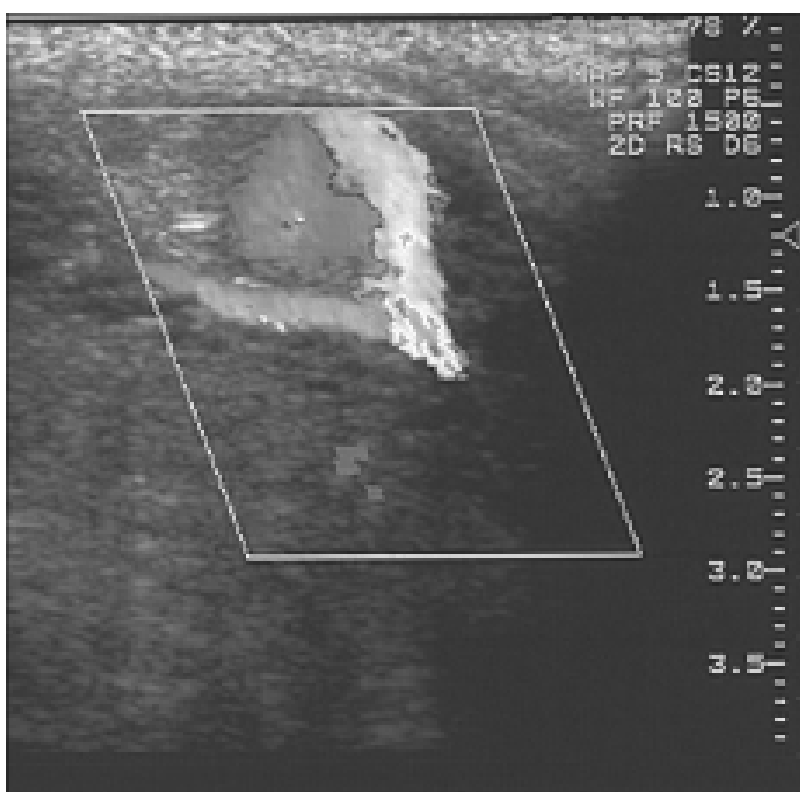

FIGURA 1. Ecografia doppler color que muestra intenso flujo arterial en raiz de ambos cuerpos cavernosos, secundario a fistula arteriocavernosa. Imagen de pseudoaneurisma.

objetiva una imagen de pseudoaneurisma en cuerpo cavernoso derecho, que desaparece tras la compresión del mismo con el transductor del ecógrafo.

Tras estos hallazgos, se procedió a la realización de una arteriografía. Por vía femoral izquierda se cateterizó la arteria iliaca primitiva contralateral, dejando colocado el extremo distal de un introductor curvo 6 Fr., inmediatamente por encima de la arteria hipogástrica. Se cateterizó selectivamente dicha arteria mediante catéter angiográfico $6 \mathrm{Fr}$. multipropósito, realizándose un estudio angiográfico en el que se demostró la existencia de fístula arteriocavernosa derecha. A través de dicho catéter, se colocó coaxialmente un microcatéter de $3 \mathrm{Fr}$., que se aproximó hasta la fístula arteriocavernosa derecha. A continuación, se realizó embolización selectiva de la fístula derecha con material reabsorbible consistente en partículas de Gelfoam (Fig. 2).

Tras la embolización de la fístula arteriocavernosa derecha, el paciente permaneció con priapismo. Se procedió a la realización de ecografía Doppler color de control, objetivándose la persistencia de fístula (Fig. 3). Posteriormente, se realizó arteriografía demostrando la existencia de fístula en lado contralateral, la cual fue embolizada 


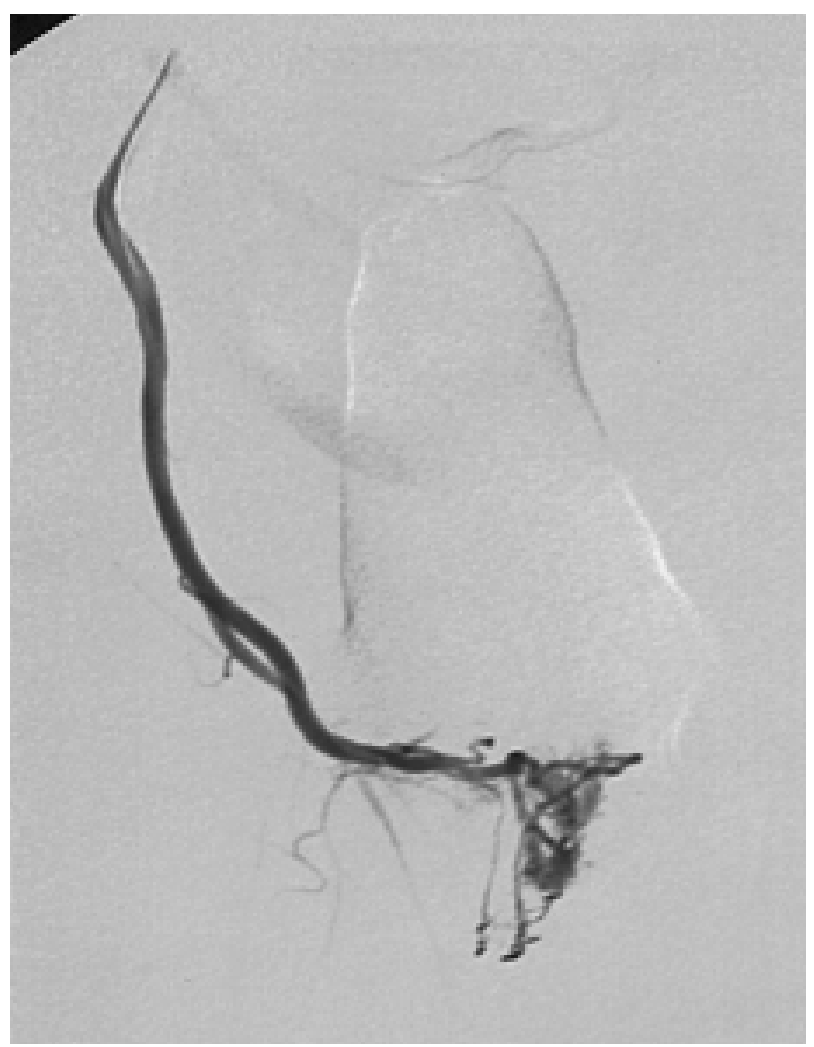

FIGURA 2. Angiografia selectiva arteria iliaca interna derecha, demostrando fistula arteriocavernosa derecha.

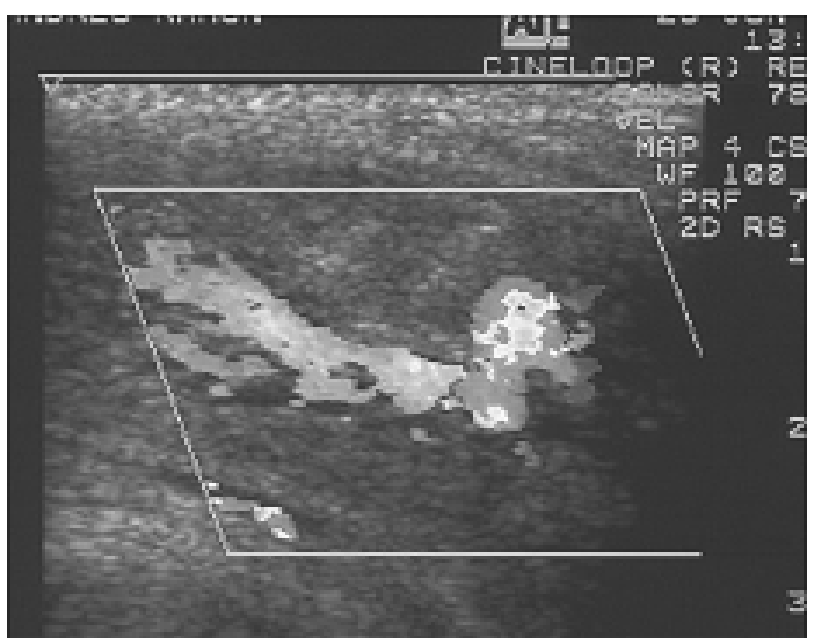

FIGURA 3. Ecografia doppler color tras embolización fistula arteriocavernosa derecha, demostrando persistencia de flujo turbulento, secundario a fistula contralateral.

en un segundo tiempo, a la semana de la primera embolización. Se procedió al abordaje por vía femoral derecha, progresando a través de hipogástrica hasta objetivarse fístula arteriocavernosa izquierda, la cual también se logró embolizar con partículas de material reabsorbible (Fig. 4).

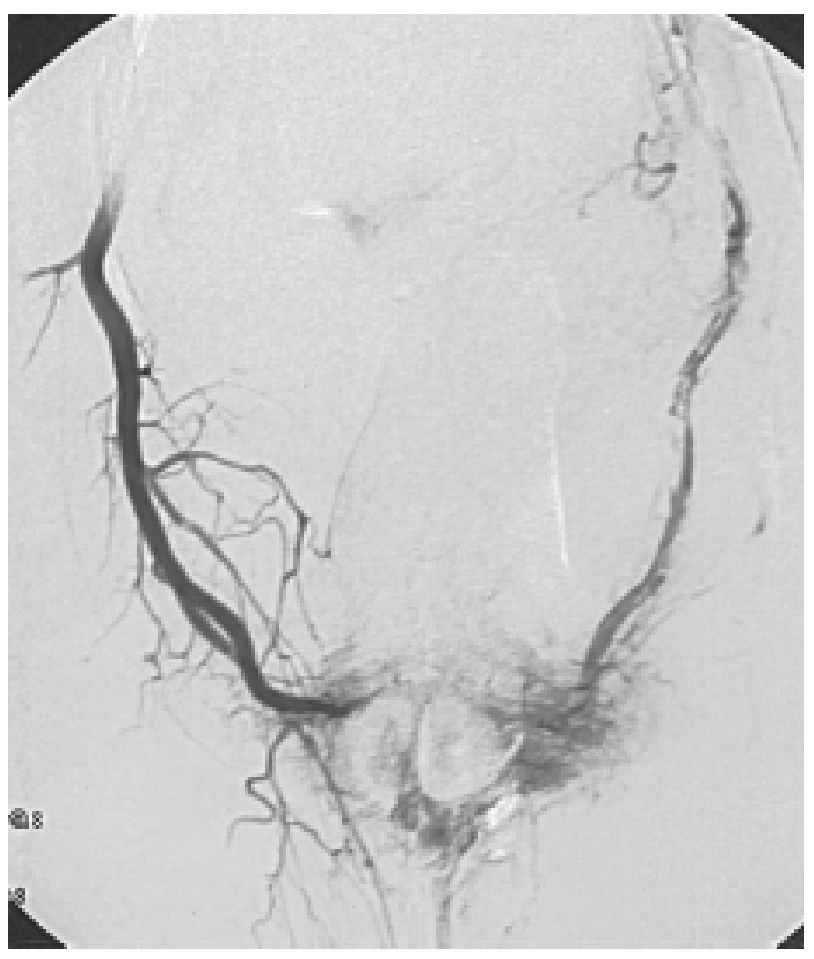

Figura 4. Angiografia selectiva sustraida arteria iliaca izquierda, objetivándose embolización de ambas fistulas.

Se produjo una detumescencia de la erección, aproximadamente a las 24 horas de evolución y, mediante una nueva imagen angiográfica, se comprobó la oclusión bilateral de ambas fístulas.

El paciente evolucionó favorablemente, con ausencia de erecciones durante aproximadamente 5 meses; tras lo cual se instauró tratamiento con citrato de sildedafilo de $50 \mathrm{mg}$, recuperando la erección fisiológica. Actualmente, el paciente consigue erecciones de buena calidad sin ayuda farmacológica.

\section{DISCUSIÓN}

El priapismo se puede definir como el estado patológico en el cual el pene se halla en erección continúa, sin tener relación con el deseo sexual. El estado de erección se limita a los cuerpos cavernosos, sin afectar al cuerpo esponjoso o el glande, como ocurre en la erección fisiológica ${ }^{1,3}$.

Esta patología se justifica por un fracaso del mecanismo de detumescencia y puede ser originado por dos causas: aumento del flujo arterial (priapismo de alto flujo o no isquémico) o disminución del retorno venoso (priapismo de bajo flujo, venooclusivo o isquémico) ${ }^{1}$. El trauma peri- 
neal cerrado o peneano son los responsables del $10-12 \%$ de los casos de priapismo, siendo la mayoría de tipo arterial ${ }^{3,5,6}$.

El priapismo arterial, entidad poco frecuente ${ }^{5}$, tiene como mecanismo fisiopatológico la laceración de la arteria cavernosa, o bien de sus ramas, con la consiguiente instauración de una fístula arteriolacunar y creación de un flujo sanguíneo turbulento en el interior de ella ${ }^{1,7}$.

La manifestación clínica característica del priapismo arterial, suele ser la latencia en la instauración ${ }^{6}$, oscilando de horas a días tras la producción del traumatismo. Esta forma de priapismo suele ser indoloro, o bien, ocasionar molestias leves, manifestándose como una erección incompleta o semirrígida, no dolorosa a la palpación ${ }^{1,3,5}$.

El diagnóstico viene hecho por el propio paciente. Son de utilidad la obtención de una gasometría intracavernosa $a^{3,4,5}$ y el estudio de flujo con ecografía Doppler color ${ }^{1-7}$, el cual delimita las situaciones de alto flujo con mejor pronóstico, de las situaciones de bajo flujo con peor pronóstico, constituyendo estás últimas una urgencia médica.

La finalidad terapéutica del priapismo arterial debe tener como meta conseguir la detumescencia y preservar la función eréctil del paciente. Aunque se han descrito casos de priapismo arterial que dejados a su evolución natural han presentado una resolución espontánea, en los casos de priapismo arterial donde se localice la fístula arteriocavernosa, la embolización selectiva transcatéter de la arteria pudenda interna con material reabsorbible como la esponja de gelatina o el coágulo autólogo ${ }^{1,3,6}$, es considerado un método seguro y efectivo para corregir el priapismo arterial postraumático, pudiendo ser el tratamiento de elección.

El tratamiento bilateral está indicado cuando el tratamiento unilateral no produce la detumescencia peneana. La embolización en dos tiempos con partículas de material reabsorbible, se postula como tratamiento electivo en los casos de priapismo arterial secundario a fístula arteriocavernosa bilateral, como el caso clínico descrito.

La detumescencia y la recuperación de erecciones de características normales es variable según las distintas series revisadas, oscilando desde días hasta 5 meses $^{1}$. En el caso descrito, el tratamiento con citrato de sildenafilo, sirvió de ayuda para la recuperación de la función eréctil. La embolización selectiva reduce el riesgo de complicaciones, preservando la función eréctil en la mayoría de los $\operatorname{casos}^{2,5,6}$.

\section{REFERENCIAS}

1. Bastuba MD, Sáenz de Tejada I, Dinlenc CZ, Sarazen A, Krane RJ, Golstein I. Arterial priapism: diagnosis, treatment and long-term followup. J Urol 1994;151(5):1231-1237.

2. Millar SF, Chait PG, Burrows PE, Steckler RE, Khoury AE, Mclorie GA, Connolly BL, Pereira JK. Posttraumatic arterial priapism in children: management with embolization. Radiology 1995;196:59-62.

3. Volkmer BG, Nesslauer T, Kraemer SC, Goerich J, Basche S, Gottfried HW. Prepuberal high flow priapism: incidence, diagnosis and treatment. J Urol 2001;166:1018-1023.

4. Hatzichristou D, Salpiggidis G, Hatzimouratidis K, Apostolidis A, Tzortzis V, Bekos A, Saripoulos D. Management strategy for arterial priapism: therapeutic dilemmas. J Urol 2002;168(5):2074-2077.

5. Park JK, Jeong YB, Han JM. Recanalization of embolized cavernosal artery: restoring potency in the patient with high flow priapism. J Urol 2001;165(6):2002-2003.

6. Langenhuijsen JF, Reisman Y, Reekers JA, De Reijke ThM. Highly selective embolization of bilateral cavernous arteries for post-traumatic penile arterial priapism. Int J Impot Res 2001;13(6):354-356.

7. Volkmer BG, Nesslauer T, Kuefer R, Kraemer S, Goerich J, Gottfried HW. High-flow priapism: a combined interventional approach with angiography and colour Doppler. Ultrasound Med Biol 2002;28(2):165-169.

Dra. I. Castaño González

Apdo. Correos 4013

35080 Las Palmas de Gran Canaria

(Trabajo recibido el 28 enero de 2004) 